\section{Hedgehog in the blood-brain barrier}

The blood-brain barrier (BBB) is partially composed of specific endothelial cells and protects the central nervous system (CNS) from pathogens and large molecules circulating in the blood. Alexandre Prat and colleagues now report that the Hedgehog signaling pathway promotes BBB formation by modulating the anti-inflammatory system (Science, published online 1 December 2011; doi:10.1126/science.1206936). The authors observed that Sonic hedgehog (Shh) was present in astrocyte-conditioned medium (ACM) but was not in endothelial cells or pericytes. They found that human Shh increased transendothelial electrical resistance (TEER) in human BBB endothelial cells and decreased permeability to large molecules. Treating these cells with cyclopamine, which antagonizes the Hedgehog pathway effector Smoothened (Smo), reversed the effects of ACM-conditioned medium. In addition, injecting mice with cyclopamine led to breakdown of the BBB in vivo. The authors constructed a conditional knockout allele that specifically removed Smo function in endothelial cells, which led to significant increases in BBB permeability. Hedgehog activation promoted quiescence of the immune system by lowering the levels of proinflammatory mediators, and the authors suggest that these new insights may lead to improvements in the delivery of drugs and therapeutics into the CNS.

\section{Intestinal stem cell interconversion}

Numerous studies have highlighted the existence of two distinct stem cell populations in the mouse intestine, one located at the crypt base and marked by expression of Lgr5 and another located at the +4 position relative to the crypt base and marked by expression of Bmi1. Jonathan Epstein and colleagues now show that these two stem cell populations share a bidirectional lineage relationship (Science, published online 10 November 2011; doi:10.1126/science.1213214). The authors identified the homeobox gene Hopx as a new marker of stem cells at the +4 position. Using lineage tracing, they showed that these Hopx-expressing cells can give rise to all cell types along the crypt-villus axis, including Lgr5-positive cells at the crypt base. In reciprocal lineage tracing studies, they found that Lgr5-expressing cells can give rise to Hopx-positive cells at the +4 position, indicating that these two stem cell populations can interconvert. The dynamics of the labeling studies indicate that the Hopx-positive cells represent a more quiescent stem cell population than the more rapidly cycling Lgr5-positive cells. These findings help reconcile prior controversies in the field and provide insights into the relationship between these two stem cell populations.

\section{Joint-rank for Mendelian sequencing}

Iuliana Ionita-Laza and colleagues report a statistical framework for exome sequencing studies used to identify the genetic basis of Mendelian disorders (Am. J. Hum. Genet., published online 30 November 2011; doi:10.1016/j.ajhg.2011.11.003). They develop a joint-rank algorithm implemented in publicly available software, which combines a filterbased approach for gene ranking, as is commonly used in current exome sequencing studies, with a new weighted-sum statistic that calculates an approximate $P$ value for each gene. Their approach allows analyses of both

Written by Orli Bahcall, Pamela Feliciano, Wayne Peng \& Kyle Vogan related and unrelated individuals within a sample. They test their method in simulations as well as on an exome sequencing dataset that included 310 unaffected individuals from an autism sequencing project. In their simulations, the joint-rank method outperformed both the filter-based and weighted-sum statistics applied individually, and the relative performance improved with increasing genetic heterogeneity. The authors also note that the performance of each method was enhanced by increasing the numbers of sequenced controls. The joint-rank application is further demonstrated on three recent exome sequencing studies for Miller, Freeman-Sheldon and Kabuki syndromes.

$O B$

\section{Lamarckian viral defense in worms}

Advances in epigenetics have begun to challenge the long-standing tenet that acquired traits are not inheritable. Now, Oliver Hobert and colleagues add viral defense in worms to the list of such Lamarckian examples and propose a mechanism independent of epigenetic modification in the germline (Cell, published online 22 November 2011; doi:10.1016/ j.cell.2011.10.042). Viral defense is mediated by virus-derived siRNAs (viRNAs), which are produced by the host RNAi machinery and silence the viral genome. The authors test the transmission of viRNA-mediated silencing and find that the antiviral response is maintained across generations; only in RNAi-defective backgrounds does the immunity taper off after four to five generations. Long-term silencing lines were established, which showed non-Mendelian inheritance that was dependent on RNAi amplification. Epigenetic modifications were ruled out as a possible mechanism because the silencing effect remained intact in chromatin modification mutants. The authors propose diffusible viRNAs as the agent of this unusual inheritance of acquired immunity. Recently, RNAi-mediated heterochromatin formation and transcriptional silencing have been reported (Nature 479, 135-139, 2011, and Nature, published online 6 November 2011; doi:10.1038/nature10492). It will be interesting to see whether these epigenetic modifications can also be maintained across generations. WP

\section{Unifying antipsychotic drugs}

The antipsychotic drugs clozapine and risperidone bind to and block the serotonin G protein-coupled receptor (GPCR) 2AR. Other serotonin antagonists, such as ritanserin and methysergide, also inhibit 2AR function, but for unknown reasons do not have antipsychotic effects. Now, Javier Gonzalez-Maeso, Diomedes Logothetis and colleagues report that signaling mediated by a heteromeric complex between 2AR and another GPCR, the metabotropic glutamate 2 receptor (mGluR2), is important for transducing the effects of antipsychotic drugs (Cell 147, 10111023, 2011). 2AR responds to the neurotransmitter serotonin and is coupled to the $G_{q} G$ protein, whereas mGluR2 responds to the neurotransmitter glutamate and is coupled to the $G_{i} G$ protein. Signaling mediated by the mGluR2-2AR heteromeric complex differs from signaling from the individual receptors. Specifically, the heteromeric complex enhances glutamate signaling through $G_{i}$ and lowers serotonin signaling through $\mathrm{G}_{\mathrm{q}}$. To quantify these signaling differences, the authors used a metric called the balance index $(B I)$, with $B I=$ change in $G_{i}$ activity $\left(\Delta G_{i}\right)$ - change in $G_{q}$ activity $\left(\Delta G_{q}\right)$. The most effective antipsychotic drugs had the highest $B I$ measurements, regardless of whether the drug targeted 2AR or mGluR2. BI measurements could also predict the psychoactive effects of a variety of compounds, suggesting that these findings may result in the identification of more effective therapeutics for schizophrenia and dementia. 